\title{
intuitio
}

Revista do PPG em Filosofia da PUCRS

http://dx.doi.org/10.15448/1983-4012.2019.1.32527

\section{ENSAIO SOBRE O CARÁTER METAFÍSICO DAS MEDIÇÕES}

\author{
ESSAY ON THE METAPHYSICAL CHARACTER OF \\ MEASUREMENTS
}

Félix Flores Pinheiro'

Resumo: O texto que segue consiste um ensaio sobre o caráter metafísico das medições, discorrendo sobre como a reflexão filosófica sobre essa atividade científica envolve elementos de discussões metafísicas. A filosofia da ciência contemporânea possui uma subárea de investigação denominada de "filosofia da medição". Tendo em vista os problemas filosóficos que emergem da prática científica, a filosofia da medição constitui um campo de estudo que investiga certos questionamentos epistêmicos, ontológicos e metafísicos sobre a natureza das medições, os quais podem ser exemplificados em três tópicos gerais: 1) em vista do que podemos medir algumas coisas e não outras; 2 ) quais condições tornam as medições possíveis; e 3) o que significam os resultados das medições. O presente texto possui por objetivo evidenciar em que sentido as investigações em filosofia da medição tocam questionamentos metafísicos. O principal argumento do presente artigo pode ser assim entendido: apesar das teorias filosóficas sobre as medições possuírem um viés epistêmico, compreender o significado das relações envolvidas nos processos de mensuração remete ao milenar problema filosófico sobre como o abstrato se relaciona com o concreto, portanto, há questões metafísicas permeando qualquer análise filosófica sobre as medições.

Palavras-chave: Metafísica. Filosofia da ciência. Filosofa da medição.

\footnotetext{
1 Universidade Federal de Santa Catarina - UFSC. Doutorando em Filosofia na UFSC (Bolsista CAPES). ORCID: http://orcid.org/0000-0001-7318-5294
} 


\begin{abstract}
The following text presents an essay on the metaphysical characteristics of measurements, in order to show how the philosophy of this scientific practice encompass metaphysical thoughts. The contemporary philosophy of science has a subarea of investigation that has been denominated by "philosophy of the measurement", which consists in a philosophical analysis that puts measurements as its ain object of study. Looking to the philosophical problems that emerge from scientific practice, the philosophy of measurement investigates some epistemic, ontological and metaphysical questions about the nature of measurements, which can be exemplified in three general topics: 1) why we can measure some things and not others; 2 ) what conditions make the measurements possible; and 3) what the results of the measurements mean The purpose of this paper is to show how the investigations in philosophy of measurement touch upon important metaphysical questions. The main argument of this article can be understood as follows: independent of the philosophical theory that we assume, any theorization about the measurements entails the ancient philosophical problem about how the abstract are related to the concrete.
\end{abstract}

Keywords: Metaphysics. Philosophy of Science. Philosophy of Measurement.

\title{
Introdução
}

Suponha que estejamos diante de uma determinada pedra de topázio² . Podemos analisar e adjetivar as características desse mineral. Diríamos que a pedra é pequena, leve, dura, fria, cara e bonita. Se nos fosse requerido algo mais específico, efetuaríamos uma avaliação comparativa, obtendo que ela é muito pequena, muito leve, pouco fria, um pouco cara e muito bonita. Porém, se quisermos ser ainda mais específicos, poderíamos expressar essa avaliação comparativa utilizando números e relações numéricas de acordo com um padrão pré-estabelecido, obtendo que (em valores aproximados): ela possui 5 centímetros de comprimento, pesa 250 gramas, sua dureza

\footnotetext{
2 Uma problematização similar é efetuada em DÍEZ, José A. A hundred years of numbers. An historical introduction to measurement theory 1887-1990: Part I: The formation period. Two lines of research: Axiomatics and real morphisms, scales and invariance. Studies in History and Philosophy of Science Part A, [s. I.], v. 28, n. 1, p. 167-185, 1997a.
} 
é 8 Mohs, sua temperatura atual está com $10^{\circ}$ Celsius, custa 200 reais e sua beleza é nível 8. Após expressarmos essas características através de números, parece que não faz sentido pensar que deveríamos ser ainda mais específicos com relação às características do objeto, porém, certamente colocar um número para expressar a beleza do objeto nos causa um certo estranhamento. Esse estranhamento está enraizado na aceitação tácita de que certas coisas são numericamente tratáveis por possuirmos procedimentos de medição para elas, enquanto outras não são tratáveis do mesmo modo. Mas, afinal, por que podemos medir algumas coisas e não outras? Além disso, mesmo que essas expressões numéricas façam sentido, o que os valores significam em diferentes casos parece variar de acordo com aquilo que foi avaliado/medido. Por exemplo, sabemos que se a pedra de topázio tem um comprimento de 5 centímetros, então ela possui o dobro do comprimento de um objeto com 2,5 centímetros e metade do comprimento de um objeto de 10 centímetros. Porém, o mesmo não pode ser dito para sua temperatura, nem para sua dureza, pois seus $10^{\circ}$ Celsius não significam o dobro de $5^{\circ}$ Celsius, nem a metade de $20^{\circ}$ Celsius $^{3}$; bem como seus 8 Mohs não significam o dobro de 4 Mohs $^{4}$. Isso significa que, enquanto podemos trabalhar com certas relações proporcionais e operações aditivas nos valores referentes ao comprimento, não podemos fazer o mesmo para outras características mensuráveis. Mas, afinal, o que determina o significado dos valores medidos? E, dando um passo atrás, que condições tornam as medições possíveis em primeiro lugar?

Ambas questões receberam a atenção de diversos filósofos no decorrer da história do pensamento ocidental. Atualmente, tais perguntas consistem em tópicos que são estudados em uma subárea da filosofia da ciência, conhecida por filosofia da medição. Resumidamente, a filosofia da

\footnotetext{
3 Esse fato é um senso comum na literatura da área e suas razões são bem conhecidas: a escala Celsius preserva apenas intervalos entre valores e seu o marco inicial, o grau o, é completamente convencional.

4 Isso ocorre porque a escala Mohs preserva apenas a ordem dos valores (isto é, não admite um tratamento de proporções numérica, tampouco preserva intervalos).
} 
medição consiste em investigações epistêmicas, metafísicas e ontológicas, com foco em problemas que emergem da prática científica das medições. Historicamente, apenas na filosofia contemporânea as medições foram colocadas como principal objeto de estudo, em especial após o trabalho de Helmholtz ${ }^{5}$, publicado originalmente em 1887. Antes disso, diversos autores teceram teses sobre a natureza das medições, mas inserindo-as em abordagens mais amplas, tratando-as de modo paralelo e/ou complementar aos principais objetos de estudo de suas investigações. Dessa forma, uma filosofia da medição pode ser vista como uma investigação datada pelo menos desde os pré-socráticos ${ }^{6}$, ou como um subcampo do saber muito recente na história do pensamento ocidental. Como resume $\mathrm{Tal}^{7}$, desde que foram tomadas como principal objeto de estudo, as medições receberam análises de cunho mais epistêmico do que metafísico, originando uma coleção vasta de publicações que podem ser organizadas em termos de escolas de pensamento. Atualmente, duas dessas escolas possuem maior influência na literatura da área, as abordagens algébricas da medição - que remontam a Helmholtz e alcançaram maturidade nas publicações da Teoria Representacional da Medição (doravante TRM) ${ }^{8}$ - e

\footnotetext{
5 HELMHOLTZ, H. Numbering and Measuring from an epistemological viewpoint. In: COHEN, Robert S.; ELKANA, Y. (ed.). Hermann von Helmholtz epistemological writings: the Paul Hertz/ Moritz Schlick Centenary Edition of 1921. [S. I.]: Springer, 1977. p. 72-102. (Boston studies in the philosophy of science, v. 37).

6 Veja-se MARI, Luca. Epistemology of measurement. Measurement, Amsterdam, v. 34, n. 1, p. 17-30, 2003.

7 TAL, Eran. Measurement in science. In: ZALTA, Edward N. (ed.). The Stanford Encyclopedia of Philosophy. Stanford: Center for the Study of Language and Information, 2015. Disponível em: https://plato.stanford.edu/entries/measurement-science. Acesso em: 04 abr. 2019.

8 Essas publicações consistem nos três volumes da obra "Foundations of Measurement": KRANTZ, David H. et al. Foundations of measurement. London: Academic Press, 1971. (Additive and Polynomial Representations, v. 1); KRANTZ, David H. et al. Foundations of measurement. London: Academic Press, 1989. (Geometrical, Threshold and Probabilistic Representations, v. 2); KRANTZ, David H. et al. Foundations of measurement. London: Academic Press, 1990. (Representation, Axiomatization and Invariance, v. 3).
} 
as abordagens baseadas em modelos ${ }^{9}$ científicos, chamadas de model-based accounts - cujo principal expoente é o próprio Tal.

Nesse cenário, o presente ensaio possui por objetivo ressaltar o caráter metafísico das medições através de três passos. Em primeiro lugar, apresentaremos o problema da natureza do que podemos medir, as chamadas grandezas, relembrando que não possuímos uma teoria metafísica que trate o assunto como principal objeto de estudo. Após isso, tendo em vista que as principais teorias da área investigam condições que tornam as medições possíveis de um ponto de vista epistêmico, discutiremos como essas abordagens remontam ao milenar problema sobre a conexão de elementos abstratos com elementos concretos. Por um lado, as abordagens matemáticas das medições afirmam a aplicabilidade do conhecimento matemático ao mundo, o que explora a relação entre entidades e relações matemáticas com entidades/propriedades e relações empíricas. Por outro lado, as abordagens baseadas em modelos científicos trabalham com a relação entre definições conceituais, como a definição de segundo padrão e procedimentos empíricos de medida, instrumentos físicos, que aproximadamente satisfazem essas definições, como relógios atômicos.

\section{A natureza das grandezas}

De um ponto de vista intuitivo, medir é efetuar um procedimento avaliativo e comparativo, não subjetivo, que possui um alvo determinado - aquilo que será medido, obtendo um resultado que usualmente consistem em um valor numérico acrescido de uma unidade de referência comparativa ${ }^{10}$. Por exemplo, medir o comprimento da minha mesa envolve

\footnotetext{
9 A concepção do que são modelos científicos está sendo trabalhada por diversos filósofos (as) contemporâneos, como em CARTWRIGHT, Nancy. The dappled world: a study of the boundaries of science. Cambridge: Cambridge University Press, 1999.

10 Como esse ensaio é voltado para as abordagens filosóficas, não discutiremos definições físicas ou matemáticas de medição. Por hora, trabalharemos com a noção intuitiva do que é
} 
encontrar um procedimento de comparação, que gera um determinado resultado, um valor para o comprimento dessa mesa específica em função da comparação realizada. Tal procedimento requer a comparação dessa característica com alguma outra coisa (o comprimento de um livro, por exemplo) e não pode ser aleatório, nem ocorrer por sorteio, a fim de obter um resultado que expresse objetivamente essa característica. Por exemplo, afirmar que a minha mesa possui aproximadamente 1,2 metros não pode ser visto como uma "medida" se eu obtive esse resultado sorteando papéis com números pré-estabelecidos e colando-os nos objetos que estão na minha sala. Isso significa que um processo de atribuição numérica conta como procedimento de medida adequado apenas se nosso método não for acidental, ou seja, ele requer alguma justificação - e isso envolve estabelecer que condições tornam uma expressão numérica objetiva e adequada, em virtude do que há procedimentos de medida válidos.

Como mostra Mari" ${ }^{1}$, determinar as condições que tornam as medições possíveis foi o objetivo de diversas investigações sobre a natureza das medições, as quais envolvem encontrar os fundamentos que tornam os processos de medida objetivos - ou seja, aquilo que distingue medições de avaliações subjetivas. Por um bom período na história da filosofia, como nos conta Kyburg ${ }^{12}$, esse fundamento foi alocado na natureza do alvo das medições. Assim, o que garante objetividade para o procedimento de medida está no modo como aquilo que está sendo medido é ontologicamente independente da subjetividade humana e acessível de modo objetivo - ou seja, possui acesso público. Esse viés origina um background realista sobre as medições, no qual a natureza daquilo que tentamos medir determina diretamente os procedimentos de medida, bem como seus resultados. Portanto, o cerne dessas investigações consiste em esclarecer em virtude

medir e, mais adiante, salientaremos algumas as concepções de medição de acordo com as teorias filosóficas que estaremos analisando.

"MARI, Luca. Epistemology of measurement. Measurement, Amsterdam, v. 34, n. 1, p. 17-30, 2003.

12 KYBURG, Henry E. Theory and measurement. Cambridge: Cambridge University Press, 1984. 
do que podemos medir algumas coisas, mas não outras, apelando para aspectos ontológicos e metafísicos das características mensuráveis.

Entender como a natureza do alvo das medições é independente dos procedimentos de medida requer entender o que são esses alvos, o que recai em uma distinção de suma importância. Seguindo o esquema utilizado por Berka ${ }^{13}$, podemos entender as medições como um processo que visa, por um lado, uma característica não particular que seja mensurável, o que chamaremos de "grandeza" e, por outro lado, o nível que objetos/eventos manifestam essas características, o que chamaremos de "magnitude". Tomando um exemplo ilustrativo, como já citado podemos medir algumas características, as grandezas, de uma pedra de topázio, como o comprimento. Porém, ao medirmos, visamos uma comparação entre a magnitude do comprimento manifestada pela pedra de topázio e a magnitude do comprimento manifestado por outro objeto.

Retomando o background realista, pensa-se que a natureza das grandezas determina a objetividade das medições, obtendo como resultado uma visão dos procedimentos de medida enquanto um processo que deve, em primeiro lugar, acessar uma grandeza e, em segundo lugar, descobrir a magnitude da grandeza em alvo. Porém, esse modo de entender como os elementos presentes nos procedimentos de medida se relacionam recai na pergunta sobre o qual é, afinal, a natureza das grandezas - o que historicamente originou uma série de posicionamentos diferentes ${ }^{14}$. Como medir envolve a comparação entre magnitudes de uma grandeza, evocando relações qualitativas entre magnitudes, e resultados que são usualmente representados através de valores numéricos e relações quantitativas, boa parte dos posicionamentos filosóficos consistiram em identificar a natureza das grandezas com um desses elementos, ou seja, afirmar que grandezas são números, ou relações numéricas, ou qualidades, ou relações qualitativas.

\footnotetext{
13 BERKA, Karel. Measurement: its concepts, theories and problems. Dordrecht: D. Reidel Publishing Company, 1983. (Boston Studies in the Philosophy of science, v. 72).

14 Veja-se KYBURG, Henry E. Theory and measurement. Cambridge: Cambridge University Press, 1984.
} 
Em geral, a identificação da natureza das grandezas com um dos elementos presentes no processo de medida afirma uma primazia desse elemento enquanto determinante para objetividade do processo. De um ponto de vista realista (e monista), afirmar essa primazia equivale a pensar o que está no mundo e determina tanto a adequação de um procedimento de medida para uma grandeza - por exemplo, em virtude do que podemos estabelecer que um certo tipo de termômetro mede a temperatura; quanto o sucesso desse procedimento em encontrar as magnitudes corretamente - por exemplo, em virtude do que avaliamos quando um termômetro específico mediu a temperatura de um líquido específico corretamente. Como já salientado, historicamente esses pensamentos se dirigiram a diversos elementos presentes nos processos de medida e podemos sintetiza-los nas seguintes teses:

1) grandezas são números e números estão no mundo, assim o procedimento de visa encontrar/descobrir uma quantidade, e graças a ela podemos derivar qualidades, como ser alto, as relações qualitativas, como ser mais alto que, e as relações quantitativas, como ser quatro vezes mais alto que...;

2) grandezas são qualidades que estão no mundo e o procedimento de medição descobre uma característica qualitativa; há algo nessa qualidade que permite derivarmos/inferirmos relações quantitativas e quantidades - atribuir números às qualidades e expressas as relações qualitativas através de relações quantitativas.

3) são as relações qualitativas que estão no mundo. Assim, por exemplo, "ser alguém alto" só faz sentido porque há em primeiro lugar a relação de "ser mais alto/baixo que" e é esse tipo de relação que determina todo o resto.

4) são as relações quantitativas que estão no mundo e medir é possível porque a natureza é composta por essas, como proporções. Assim, "ser mais alto que" só faz sentido porque há em 
primeiro lugar proporções no mundo, o que nos permite valorar corretamente cada participante dessa relação, preservando-a, e derivando qualidades e relações qualitativas.

Entendido isso, cabe ressaltar que esse debate está completamente em aberto na literatura da área. Como as medições foram colocadas enquanto principal objeto de estudo apenas recentemente, possuímos poucas teorias maduras sobre o assunto, em especial nos termos de um tratamento metafísico sobre a natureza das grandezas. Nesse sentido, salientamos que está disponível para autores interessados nesse tópico prosseguir com a defensa de qualquer uma das posturas acima. Podemos seguir uma tradição filosófica que remonta ao menos aos pitagóricos e construir argumentativamente uma defesa de 4), ou seguir autores como Michell ${ }^{15}$ e ofertar uma defesa de um realismo de entidades matemáticas, como em 1). Podemos pensar algo no sentido de 2), que grandezas são certos tipos de qualidades, como Campbell ${ }^{16}$ nos lega, ou defender 3 ), de modo similar ao que foi feito por Helmholtz, argumentando que que relações qualitativas são os elementos determinantes para os procedimentos de medição. Por fim, podemos construir uma visão teórica que recuse a primazia colocada nos termos de 1) - 4) - o que pode ser feito tanto adotando uma visão metafísica pluralista (ou seja, pensando que as grandezas não se esgotam em um único tipo de coisas); quanto recusando um viés epistêmico fundamentalista, em prol de uma visão coerentista dos procedimentos de medição, algo similar ao realizado por Chang ${ }^{17}$.

De qualquer modo, o debate está em aberto especialmente por conta de certas dificuldades teórico-metodológicas que qualquer teoria sobre a natureza das grandezas enfrenta. Tendo em vista que medições são

15 MICHELL, J. Making the representational theory of measurement. In: MICHELL, Joel. Measurement in psychology: a critical history of a methodological concept. Cambridge: Cambridge University Press, 1999. p. 109-139.

${ }^{16}$ CAMPBELL, Norman Robert. Physics: the elements. London: Cambridge University Press, 1920.

17 CHANG, Hasok. Inventing temperature: measurement and scientific progress. Oxford: Oxford University Press, 2007. (Oxford Studies in Philosophy of Science). 
atividades ubíquas, mas não unívocas, possuímos concepções intuitivas e culturais que nos servem de critérios para avaliar o quão correto e completo é o escopo de uma teoria metafísica sobre elas, isto é, como os critérios que a teoria utiliza para classificar o que é uma "grandeza" captura tudo e apenas aquilo que é mensurável. Dessa forma, a principal dificuldade para uma metafísica monista das grandezas está em fornecer uma elucidação geral também sirva como critério para separar o que pode ser alvo de uma medição, do que não pode.

A fim de ilustrar tamanha diversidade, de um ponto de vista intuitivo as grandezas possuem três peculiaridades: i) elas são acessíveis; ii) suas manifestações admitem níveis, isto é, faz sentido em falar na magnitude delas; iii) esses graus não são infinitos (respeitam o princípio de Arquimedes) ${ }^{18}$. Tais características são apresentadas e discutidas na literatura da área, mas podemos elucidá-las através de crenças comuns sobre as medições, por exemplo, que só podemos medir aquilo que nossos procedimentos de medições acessam, que medir envolve comparar os níveis ou graus de exibição de algo (uma relação de "semelhante ou maior ou menor que") e que manifestações infinitas não são empiricamente detectáveis/mensuráveis. Porém, mesmo essas caracterizações mais gerais não encontram consenso na literatura filosófica sobre o tema, em especial o ponto iii) ${ }^{19}$. Ainda assim, as caracterizações i), ii) e iii) nos são úteis para exemplificar a diversidade das coisas que podemos medir, ou seja, as grandezas. Se pensarmos que elas formam um conjunto de condições necessárias para algo ser uma grandeza, mesmo que provisórias e revisáveis, temos um

18 O princípio de Arquimedes é discutido nas abordagens algébricas da medição, usualmente formulado enquanto axioma. Grosso modo, o princípio de Arquimedes aplicado ao contexto das medições diz que, se um objeto $A$ é maior do que um objeto $B$, existe um determinado número $n$ (usualmente um número natural) de vezes em que a grandeza de $B$ alcança ou ultrapassa a grandeza de A: se A > B, então $(n) B \geq A$. Veja-se SUPPES, Patrick. A set of independent axioms for extensive quantities. Portugaliæ Mathematica, Lisboa, v. 10, n. 4, p. 163-172, 1951.

19 Um exemplo interessante dessa abordagem está no trabalho de NARENS, Louis. Abstract measurement theory. Cambridge: MIT Press, 1985; o qual discute sistemas algébricos que não recorrem ao princípio de Arquimedes. 
teste para determinar o que em princípio não podemos medir: se algo não satisfaz i), ii) ou iii) esse algo não é mensurável. Isso pode ser compreendido através de um exemplo ilustrativo, como por exemplo, questionando se podemos medir o amor. Tendo em vista i), o amor pode ser uma grandeza se o entendermos enquanto uma manifestação que pode ser detectada, caso contrário não poderíamos medi-lo. Através de ii), entende-se que além de poder ser detectado, deveríamos admitir que o amor se manifesta em diferentes magnitudes, fazendo sentido falar em "amar/ser amado mais ou menos que...". Por fim, esses níveis de "amar/ser amado" não podem ser infinitos para serem mensurados, respeitando iii). Assim, se concordarmos que o "amor" pode ser detectado, admite graus e esses são finitos, então, em princípio, poderíamos ter medidas para o "amor". Por outro lado, se recusarmos qualquer uma dessas concepções, então o "amor" não pode ser visto como uma grandeza. Por exemplo, poderíamos recusar ii) pensando que as manifestações de amor não admitem graus.

Como consequência de adotar uma definição de "grandeza" através dessas condições, obtemos que características mensuráveis possuem uma natureza diversa, pois características naturais satisfazem essas condições, mas também conceitos de ordem social e econômica, psicológica e subjetiva. Para citar exemplos, utilizando i), ii) e iii) temos que a temperatura, o peso/a massa, a velocidade, o comprimento, a duração, a dureza, a solubilidade, a vazão, dentre outros, são características em princípio mensuráveis, enquanto outras características naturais não são grandezas por ferirem uma dessas condições, como ser um cachorro, ou ser careca (visto que não faz sentido pensar que um animal é "mais cachorro" que outro, ou que um ser humano é "mais careca"). Além disso, obtemos características econômicas e sociais, como ser rico, ser solteiro, a inflação, ser caro (o valor de mercado/preço); em detrimento de características como ser a moeda de um país, ser um cidadão ou ser casado, que ferem ii). Por fim, conseguimos aplicar esse teste para distinguir grandezas e "não-grandezas" de cunho psicológico e subjetivo, pois, por exemplo, a sensação de dor, o estresse e a beleza, satisfazem 
i), ii) e iii), em detrimento do sentimento de indiferença, para o qual ii) não se aplica. Nesse sentido, as condições i), ii) e iii) nos oferecem um recorte para as coisas que não são grandezas em variados âmbitos, nos ajudando a agrupar características mensuráveis em tipos: naturais, econômico-sociais, psicológicas e subjetivas.

Essas considerações são importantes, em primeiro lugar, porque é tarefa de uma investigação sobre a natureza daquilo que podemos medir fornecer e esclarecer o que são grandezas, mas também mostrar em que sentido elas se relacionam umas com as outras, originando uma taxonomia. Em segundo lugar, é importante perceber essa variedade por ser comum encontrarmos esses tipos misturados na literatura da área ${ }^{20}$. Nesse sentido, uma teoria que possua por objetivo esclarecer em virtude do que podemos medir algumas coisas e não outras focando nas condições que tornam algo mensurável, desde um ponto de vista da natureza das grandezas, esbarra na dificuldade de fornecer um conjunto de elementos que essas possuem em função de sua diversidade. Atualmente, além das teorias sobre o assunto que encontramos na literatura da área, dispomos de dois tipos de ferramentas para trabalhar a natureza desses conceitos, as quais nos servem enquanto estratégias para lidar com esse tema em estudos futuros e construir novas abordagens. A primeira consiste em uma análise sobre os comprometimentos ontológicos das teorias que lidam com conceitos mensuráveis, entendida como uma investigação de ontologia analítica ${ }^{21}$, ou ontologia "naturalizada". A segunda, uma análise conceitual que construa uma teoria metafísica em sentido "tradicional”22. Como veremos, ambas

\footnotetext{
${ }^{20}$ Diversos estudos em filosofia da medição teorizaram tanto sobre grandezas naturais, quanto psicológicas e econômicas. Inclusive, a utilização de dados quantitativos nos campos da psicologia certamente impulsionou as principais teorias do campo, como a Teoria Representacional da Medição.

21 Veja-se KRAUSE, Décio. Tópicos em ontologia analítica. São Paulo: UNESP, 2017.

22 A distinção entre abordagens entendidas como "ontologia naturalizada" e "metafísica tradicional" utilizada aqui, foi apresentada em ARENHART, Jonas R. B. Ontological frameworks for scientific theories. Foundations of Science, [s. I.], v. 17, n. 4, p. 339-356, 2012.
} 
esbarram no mesmo desafio: ter a completude do escopo conceitual adequado às nossas intuições comuns sobre o que é medir.

Por um lado, poderíamos adotar um viés monista e utilizar como estratégia uma análise nos termos da ontologia naturalizada, construindo abordagens que evidenciem os comprometimentos ontológicos do tratamento científico das grandezas, observando a adequação do escopo da teoria em relação à prática científica. Por exemplo, se a partir dessa análise obtivermos que as grandezas devem ser entendidas enquanto estruturas relacionais ${ }^{23}$, como na prática científica medimos a velocidade, então a velocidade tem de ser um conceito tratável através dessas estruturas, ou caso não for, devemos encontrar nessa abordagem alguma ressalva elucidativa a esse respeito. O mesmo vale para uma abordagem através da metafísica tradicional, pois se visarmos elucidar o que são as grandezas através de conceitos como "acidentes", ou "qualidades" (primárias ou secundárias), ou "capacidades", ou "propriedades emergentes", dentre outros, também esbarramos no problema do escopo. Ademais, além de parecer difícil encontrar um único conceito para a classe daquilo que é mensurável, também nos é exigido salientar o que torna a classe das grandezas distinta de outras coisas que cabem no mesmo conceito. Exemplificando, se defendermos que grandezas são capacidades, deveríamos explicitar se, de fato, todas as grandezas são capacidades e se todas as capacidades são grandezas, e se não for o caso isso não ocorrer, retomar a explicação do que, afinal, torna algo uma grandeza. Por outro lado, assumindo uma postura pluralista, ambas abordagens possuem liberdade para trabalhar com duas ou mais caracterizações, possuindo o mesmo desafio de esclarecer em virtude do que podemos ter, se é que podemos, grandezas que são, por exemplo, qualidades de primeira e de segunda ordem e qualidades de primeira e de segunda ordem que não são grandezas - ou seja, mostrar o que as distinguem.

23 Esse conceito deriva da abordagem da TRM e será esclarecido adiante 
Tais estratégias compõem uma coleção de estudos em aberto e promissores disponíveis para filósofos que estejam interessados nos elementos metafísicos advindos da prática das medições. Muito desse caráter em aberto sobre a natureza das grandezas deriva do fato da literatura da área, a filosofia da medição, ter sido construída no espírito de uma investigação mais epistêmica do que metafísica ${ }^{24}$. De acordo com os historiadores da área ${ }^{25}$, a filosofia da medição contemporânea é marcada pelas investigações que colocaram as medições como principal objeto de estudo, remontando tradicionalmente ao estudo de Helmholtz. Como o autor afirma, até aquele momento, "embora numerar e medir sejam os alicerces dos métodos científicos mais frutíferos, seguros e exatos que conhecemos, relativamente pouco trabalho foi feito nas suas bases epistemológicas ${ }^{26}$, iniciando um estudo sobre as condições que tornam características empíricas mensuráveis. $\mathrm{Na}$ esteira dessa abordagem, passados mais de um século desde a publicação desse ensaio, possuímos como legado uma teoria madura sobre o assunto - a TRM, além de diferentes escolas de pensamento sobre o caráter epistêmico das medições. Tais escolas se distinguem pelo modo como pensam o que é "medir", dentre as quais se destacam a escola da abordagem algébrica das medições (que inclui a teoria supracitada) e as abordagens baseadas em modelos científicos.

Reconhecendo que a prática das medições científicas envolve uma série de convenções, podemos questionar em virtude do que as medições se distinguem de avaliações subjetivas e puramente convencionais

\footnotetext{
24 Uma exceção notável a isso é o trabalho de MICHELL, J. Making the representational theory of measurement. In: MICHELL, Joel. Measurement in psychology: a critical history of a methodological concept. Cambridge: Cambridge University Press, 1999. p. 109-139.

${ }_{25}$ Veja-se DíEZ, José A. A hundred years of numbers. An historical introduction to measurement theory 1887-1990: Part I: The formation period. Two lines of research: Axiomatics and real morphisms, scales and invariance. Studies in History and Philosophy of Science Part A, [s. l.], v. 28, n. 1, p. 167-185, 1997 a.

${ }^{26}$ HELMHOLTZ, H. Numbering and Measuring from an epistemological viewpoint. In: COHEN, Robert S.; ELKANA, Y. (ed.). Hermann von Helmholtz epistemological writings: the Paul Hertz/ Moritz Schlick Centenary Edition of 1921. [S. I.]: Springer, 1977. p. 72-102. (Boston studies in the philosophy of science, v. 37).
} 
- fornecem conhecimento em sentido objetivo. Observando as teorizações sobre o tema temos, por um lado, uma abordagem fundacionalista, advinda dos tratamentos algébricos da medição, em especial da Teoria Representacional da Medição. Por esse viés, há relações empíricas qualitativas e observáveis que devem satisfazer uma série de condições suficientes para que possamos atribuir números aos objetos (ou às qualidades dos objetos), possibilitando efetuarmos uma medição que não dependa de valores assumidos previamente. Por outro lado, encontramos um viés coerentista nas abordagens baseadas em modelos, as quais reconhecem que medir envolve a construção de um experimento, ou instrumento, bem como de escalas, o que pode pressupor entendimentos advindos de teorias científicas - inclusive utilizando valores previamente estabelecidos por constantes físicas. De acordo com essa abordagem, medir envolve correlacionarmos a prática científica com conceitos e partes de teorias científicas, formando um círculo virtuoso (não vicioso) por conta dessa correlação ser fonte de progresso científico ${ }^{27}$.

Ainda que o principal enfoque dessas investigações esteja voltado para uma epistemologia científica, há elementos metafísicos latentes em ambas, remontando milenar problema filosófico sobre como o abstrato se relaciona com o concreto. Apesar dessa pergunta pairar no horizonte de qualquer teorização sobre o mundo físico, ela se mostra com destaque ímpar nas medições. Isso ocorre porque, como veremos, os modos como entendemos as medições - cuja definição varia de acordo com a teoria utilizada - trabalham diretamente com a conexão entre objetos, instrumentos, esquemas e conceitos, pois independentemente da teoria utilizada, medir envolve primordialmente a construção de relações entre o mundo empírico, conceitos físicos e e o conhecimento matemático. No que segue, veremos como isso ocorre a partir do esquema geral da

${ }_{27}$ Veja-se TAL, Eran. Old and new problems in philosophy of measurement. Philosophy Compass, [s. l.], v. 8, n. 12, p. 1159-1173, 2013. 
abordagem fomentada pela TRM e da abordagem baseada em modelos, respeitando as peculiaridades de cada uma delas.

\section{As medições e a aplicabilidade do conhecimento matemático}

A TRM é entendida, de um ponto de vista histórico, como a culminância de duas abordagens sobre as medições ${ }^{28}$. Por um lado, os trabalhos já mencionados de Helmholtz e Campbell visaram encontrar as características empíricas que tornam uma medição possível, entendendo-a enquanto um modo de assinalar objetivamente números ao mundo. Por outro, os trabalhos de Stevens ${ }^{29}$ analisaram as características algébricas das escalas, pensando as medições através de modos de assinalar números de acordo com uma regra. Tais estudos paralelos são unificados pela primeira vez no artigo de Suppes ${ }^{30}$, formando uma teoria madura sobre o assunto após uma série de trabalhos colaborativos, os quais foram organizados e publicados nos três volumes de "Foundations of Measurement"31.

Grosso modo, tendo a teoria intuitiva de conjuntos como framework, essa abordagem estabelece que medir constitui construir um "mapeamento" entre dois tipos de estruturas relacionais, qualitativas e quantitativas, o que nesse caso significa estabelecer um homomorfismo entre ambas. Esse mapeamento pode ser entendido através de 3 etapas, possuindo uma etapa prévia. Segundo os expoentes da TRM, medir algo envolve

\footnotetext{
${ }_{28}$ Veja-se DíEZ, José A. A Hundred Years of Numbers. An historical introduction to measurement theory 1887-1990: Part 2: Suppes and the mature theory. Representation and uniqueness. Studies in History and Philosophy of Science Part A, [s. l.], v. 28, n. 2, p. 237-265, 1997b.

29 STEVENS, S. S. On the theory of scales of measurement. Science, [s. l.], v. 103, n. 2684, p. 677-680, June 1946.

30 SUPPES, Patrick. A set of independent axioms for extensive quantities. Portugaliæ Mathematica, Lisboa, v. 10, n. 4, p. 163-172, 1951.

31 KRANTZ, David H. et al. Foundations of measurement. London: Academic Press, 1971. (Additive and Polynomial Representations, v. 1). KRANTZ, David H. et al. Foundations of measurement. London: Academic Press, 1989. (Geometrical, Threshold and Probabilistic Representations, v. 2). KRANTZ, David H. et al. Foundations of measurement. London: Academic Press, 1990. (Representation, Axiomatization and Invariance, v. 3).
} 
estabelecer procedimentos empíricos - como a comparação de objetos de acordo com a magnitude da grandeza visada, estabelecendo se esse nível é "semelhante", "maior ou menor que"; e um procedimento de combinação, ou concatenação empírica, para a magnitude da grandeza desses objetos. Após isso, representamos os objetos que exibem a grandeza em alvo, bem como suas relações empíricas observáveis através dos resultados dos procedimentos de combinação e concatenação, em uma estrutura de relações qualitativas, definidas do seguinte modo:

Def. 1. Uma estrutura $E=<D, R_{1}, \ldots R_{n}>$ é uma estrutura de relações qualitativas, se possui um domínio $D$ não vazio de elementos (não numéricos) e pelo menos uma relação $R_{j}\left({ }_{j}=1, \ldots, n\right)$ n-ária entre os elementos de $D$.

Nesse sentido, para medirmos, por exemplo, o comprimento, precisamos observar o comportamento de relações qualitativas próprias dessa grandeza, estabelecendo, se possível, um procedimento de comparação e um procedimento de concatenação para suas magnitudes. Feito isso, representaremos os objetos e o comportamento dessas relações em uma estrutura relacional qualitativa, fornecendo axiomas para as relações $<R_{1}$, $\ldots R_{n}>$, a fim de que essas "espelhem" o resultado dos procedimentos empíricos. Por exemplo, concatenar dois objetos de acordo com o comprimento sempre resulta em um comprimento maior do que o exibido pelas suas partes, o que pode ser expressado através de um "axioma da positividade" (abreviando "a maior que b" por "a > b" e "a está concatenado com b" por "a*b"): $\neg(a>(a * b))$. Cabe ressaltar, portanto, que cada grandeza em alvo pode ser representada em uma estrutura de relações qualitativas, visto que as relações empíricas observáveis delas se comportam de maneiras diferentes, originando uma coleção de axiomas diferentes ${ }^{32}$.

32 Isso ocorre de modo muito interessante, pois gera diferentes estruturas apenas para diferentes classes de grandezas. Por exemplo, grandezas ditas "extensivas", como o peso e o comprimento, podem ser representadas em uma mesma estrutura relacional qualitativa. Mas 
Por trabalhar na teoria intuitiva de conjuntos, a abordagem tem à disposição um segundo tipo de estrutura relacional, as quantitativas (numéricas), assim definidas:

Def. 2. Uma estrutura $E^{\prime}=\left\langle D^{\prime}, R_{j}\right\rangle$ é dita uma estrutura relacional quantitativa se $D^{\prime}$ é um subconjunto não vazio dos números reais e cada $\operatorname{Rj}^{\prime}(j=1, \ldots, n)$ for uma relação de aridade $n$ entre os elementos de $D^{\prime}$.

Diante disso, a teoria estabelece que medir é mapear uma estrutura qualitativa em uma estrutura quantitativa, com relações de mesma aridade, atribuindo números aos elementos do domínio qualitativo e preservando as relações (obtendo um homomorfismo). Esse "mapeamento" é feito a partir de três passos. Em primeiro lugar, lançamos como hipótese que há uma função mapeamento, a qual é entendida do seguindo modo:

Def. 3. Uma função $f$ de $D$ em D' é dita uma função mapeamento se constrói um homomorfismo de $\mathrm{E}$ em E'.

A partir disso, o segundo e terceiro passos consistem na prova de dois teoremas, o teorema de representação e o teorema de unicidade. No esquema geral da TRM, o teorema de representação cumpre os papéis de provar que existe pelo menos uma função mapeamento para a estrutura relacional qualitativa em foco, e de nos fornecer a garantia de que, quando o provamos, há uma representação numérica adequada para essa estrutura. Informalmente, o teorema pode ser entendido como a prova da condicional: se a estrutura relacional qualitativa satisfaz certas condições - que nesse caso são os axiomas que determinam o comportamento das relações qualitativas $\mathrm{Rj}$ - então existe uma função mapeamento para ela. 
Porém, como podemos obter diferentes funções mapeamento para a mesma estrutura qualitativa, o teorema de unicidade estabelece o que todas essas funções, e assim todas as representações adequadas, possuem em comum. A partir desse segundo teorema, provamos que as diferentes representações adequadas para uma mesma estrutura qualitativa são todas de um mesmo tipo através das transformações algébricas que as funções mapeamento admitem, isto é, se $f$ e $g$ são funções mapeamento para uma mesma $E$, então existe uma função $h$, tal $g=h^{\circ} f$, sendo "0" um símbolo para a composição de funções. Como mostra Díez ${ }^{33}$, podemos agrupar ambos teoremas em um só:

Teorema de representação-unicidade: Seja $E=<D, R j>$ uma estrutura qualitativa e $E^{\prime}=\left\langle D^{\prime}\right.$, Rj' $\rangle$ uma estrutura quantitativa, se E satisfaz os axiomas ( $A x-A x+n)$, então existe uma função $f$ de $D$ em $D^{\prime}$ tal que, para qualquer outra $g, g$ é um homomorfismo de $E$ em E' se, e somente se, $g$ é uma T-transformação de $f$.

Sintetizando, de acordo com a TRM medir é construir uma representação numérica através de um processo de "mapeamento" de uma estrutura de relações qualitativas em uma estrutura de relações numéricas. Essa teoria constituiu uma espécie de paradigma na literatura da área até, pelo menos, a década de 2000 . Porém, nas últimas duas décadas, ela tem recebido uma série de críticas. Certamente, uma das principais críticas dirigidas à abordagem algébrica das medições está na constatação de que a mesma é demasiado abstrata, distante da prática experimental científica ${ }^{34}$. Isso ocorre porque grande parte dos axiomas administrados pela teoria

33 DÍEZ, José A. A Hundred Years of Numbers. An historical introduction to measurement theory 1887-1990: Part 2: Suppes and the mature theory. Representation and uniqueness. Studies in History and Philosophy of Science Part A, [s. l.], v. 28, n. 2, p. 237-265, 1997 b.

34 Veja-se FRIGERIO, Aldo; GIORDANI, Alessandro; MARI, Lucas. Outline of a general model of measurement. Synthese, Dordrecht, v. 175, n. 2, p. 123-149, July 2010. 
como condições que devem ser satisfeitas para que possamos construir um mapeamento não fazem sentido desde um ponto de vista empírico.

Diante dessas críticas, autores como Heilmann ${ }^{35}$ sugeriram que a Teoria Representacional da Medição merecia uma atualização, fomentando uma nova interpretação para a mesma. Segundo ele, a teoria deve ser vista como uma ferramenta, um método para relacionar concepções qualitativas com concepções quantitativas, cujo corpo teórico consiste em uma coleção de teoremas. Assim, o autor nos lembra que o caráter da teoria é fundamentalmente matemático, o que é facilmente compreendido visto que a mesma surge da continuidade de um programa, iniciado por Helmholtz, voltado para a aplicabilidade do conhecimento matemático ao mundo ${ }^{36}$.

Porém, na tentativa de cumprir a tarefa de responder como nós podemos representar a natureza quantitativamente, a teoria utiliza a noção de estrutura relacional qualitativa para fazer uma ponte entre um campo matemático e um campo empírico. Nesse sentido, nós não atribuímos números diretamente aos objetos (ou às suas qualidades), mas às estruturas relacionais qualitativas que os representam. Todavia, pouco trabalho é feito nos termos de justificar como, afinal, passamos de relações empíricas para seus representantes, as relações qualitativas condicionadas por axiomas.

Segundo seus expoentes:

Enfatizamos que as escalas numéricas de medições estão sujeitas a convenções arbitrárias. Existem transformações admissíveis, que correspondem a escolhas de unidade, e os próprios teoremas de representação e de unicidade dependem da escolha convencional de uma estrutura numérica

\footnotetext{
35 HEILMANN, Conrad. A new interpretation of the representational theory of measurement. Philosophy of Science, Baltimore, v. 82, n. 5, p. 787-797, Dec. 2015.

36 Um panorama histórico do tratamento da aplicabilidade do conhecimento matemático realizado por autores da abordagem algébrica das medições pode ser encontrado em CANTÙ, Paola. The epistemological question of the applicability of mathematics. Journal for the History of Analytical Philosophy, [s. l.], v. 6, n. 3, p. 95-114, 2018.
} 
relacional. O que é invariante e, portanto, não é uma questão de convenção, é a estrutura relacional empírica e suas propriedades empíricas, algumas das quais são formuladas como axiomas. Uma coleção de axiomas que guiam os teoremas da medição fundamental pode ser entendida como um conjunto de leis empíricas qualitativas (isto é, não numéricas) ${ }^{37}$.

Pensar que o fornecimento de axiomas consiste em estabelecer leis com conteúdo empírico é algo demasiado controverso, visto que boa parte deles simplesmente não fazem sentido desde um ponto de vista da prática experimental das medições. Esse ponto é reconhecido tanto pelos autores que criticaram a abordagem, quanto pelos seus próprios expoentes. Uma vez que os axiomas formam condições para que uma estrutura seja numericamente representável, tais condições deveriam ser suficientes e/ou necessárias. Todavia, o caráter de condições suficiente ou condição necessária para as estruturas não advém de qualquer consideração empírica - sobre aquilo que a estrutura qualitativa supostamente representa. Como os próprios autores afirmam, ser condição necessária:

significa necessidade matemática, não prática. Um axioma é necessário se ele é uma consequência da existência do homomorfismo que estamos tentando estabelecer [...] não possuímos quaisquer regras para selecionam o conjunto correto de axiomas necessários; em geral, é uma questão de tentativa e erro ${ }^{38}$.

Do nosso ponto de vista, como os axiomas são estabelecidos através de "tentativa e erro", seu conteúdo empírico é colocado em segundo plano, em função de um sucesso determinado pelos elementos formais do mapeamento.

\footnotetext{
37 KRANTZ, David H. et al. Foundations of measurement. London: Academic Press, 1971. (Additive and Polynomial Representations, v. 1), p. 13.

38 KRANTZ, David H. et al. Foundations of measurement. London: Academic Press, 1971. (Additive and Polynomial Representations, v. 1), p. 22.
} 
Nesse sentido, mesmo se supormos, para fins de discussão, que a Teoria Representacional da Medição estabelece um bom modo de relacionarmos elementos qualitativos com elementos quantitativos, a mesma nos deixa em dúvida sobre como, afinal, a natureza permite uma representação axiomática. Questionar isso significa pôr em dúvida justamente a aplicabilidade da ferramenta, do método afirmado pela teoria: o que nos legitima a estabelecer que relações empíricas possam ser representadas através de condições axiomáticas? Assim, há um elemento metafísico que é de suma importância, o qual reside na afirmação da aplicabilidade do conhecimento matemático ao mundo, remontando ao clássico problema sobre como relações empíricas estão conectadas com relações algébricas (formais, teóricas e abstratas).

\section{A “realização" de definições idealizadas}

Boa parte das críticas à Teoria Representacional da Medição surgiram de autores que defendem uma model-based accounts das medições. Seguindo essa escola de pensamento, medir envolve muito mais do que analisar as propriedades algébricas das escalas e de modo algum se esgota na ideia de construção de um “mapeamento" entre estruturas, sendo que "esse modo de conceber a medição conduz a subestimar problemas importantes tanto na teoria como na prática das medições"39.

Grosso modo, a partir de um viés pragmatista, essa abordagem parte do reconhecimento de que medir algo, na atividade científica, envolve a construção de um experimento que pressupõe certo conhecimento sobre a grandeza em alvo, o qual advém de teorias científicas sobre o assunto. Como já afirmamos, essa postura consiste em um viés coerentista sobre as medições, assumindo que as questões "o que conta como procedimento válido para medir x" e "o que é x" não fazem sentido separadas - isto é, não

39 FRIGERIO, Aldo; GIORDANI, Alessandro; MARI, Lucas. Outline of a general model of measurement. Synthese, Dordrecht, v. 175, n. 2, p. 123-149, July 2010. 
podem ser respondidas afirmando a primazia de uma em detrimento da outra ${ }^{40}$. Por exemplo, para medir a massa de um objeto precisamos construir um instrumento de medição e isso só é possível se tivermos algum conhecimento prévio sobre o que é a massa. Ao mesmo tempo, essa abordagem reconhece que as teorias não são facilmente aplicáveis e a construção de um experimento envolve dificuldades práticas que ultrapassam o escopo teórico dessas, exigindo inclusive a consideração de partes de duas ou mais teorias diferentes. Assim, a construção de um instrumento de medição é mediada por um modelo: um constructo esquemático, abstrato, simplificado e local, com uma função explícita e bem delimitada ${ }^{41}$.

As abordagens baseadas em modelos afirmam que medir envolve procedimentos em dois níveis: um teórico e abstrato, e um físico e instrumental. Segundo eles, o nível físico consiste nas operações empíricas que subjazem a construção de um procedimento de medida. Esse nível é guiado pelo âmbito abstrato, que consiste nos modelos teóricos e estatísticos do processo, o qual fornece diretrizes para construir o experimento, bem como para interpretar os resultados empíricos que os experimentos originam, estabelecendo regras de inferências válidas e margens de erro. Assim, os modelos cumprem as funções de definir os parâmetros mensuráveis, construir as escalas, direcionar o design dos instrumentos e a preparação para o experimento, auxiliar a detecção de erros e estimar incertezas, e, por fim, direcionar as inferências válidas na interpretação dos outcomes empíricos fornecidos pelos instrumentos físicos. Diante disso, de acordo com essa abordagem, medir é interpretar os resultados físicos de um experimento no modelo.

\footnotetext{
40 Esse tópico é aprofundado em CHANG, Hasok; CARTWRIGHT, Nancy. Measurement. In: PSILLOS, Stathis; CURD, Martin (ed.). The Routledge companion to philosophy of science. 2nd ed. New York: Routledge, 2013. p. 411-419.

${ }_{41}$ Veja-se o tópico 7 em TAL, Eran. Measurement in science. In: ZALTA, Edward N. (ed.). The Stanford Encyclopedia of Philosophy. Stanford: Center for the Study of Language and Information, 2015. Disponível em: https://plato.stanford.edu/entries/measurement-science. Acesso em: 04 abr. 2019.
} 
Resumidamente, esse procedimento de construção de modelos e instrumentos pressupõe conhecimentos advindos de diversas teorias científicas, fornecendo uma abordagem coerentista sobre a construção de conhecimento científico, a qual afirma dois tipos de coerência. A primeira, entre modelo construído e teorias de fundo, pois é a teoria que determina quando e se o que se quer medir está sendo alcançado. A segunda, entre os resultados obtidos através de diferentes modelos para medir a mesma grandeza. De acordo com isso, a objetividade do processo recai em mostrar que os resultados obtidos são independes dos indivíduos, e do ambiente, que realizam os experimentos. Por exemplo, que o resultado não é fruto do interesse de um sujeito, ou de alguma aleatoriedade presente no ambiente em que o experimento é realizado. Por consequência, esse esquema para as medições cumpre o propósito de mostrar que os experimentos não têm uma dependência do contexto que comprometa a objetividade dos seus resultados, validando suas aplicabilidades gerais.

Todavia, o mesmo tipo de consideração que foi direcionado para as teorias algébricas da medição pode ser feito aqui. Naquele caso, a teoria oferece as estruturas qualitativas relacionais como ponte entre um âmbito completamente empírico e um âmbito abstrato, matemático. Nesse caso, a abordagem oferece os modelos como ponte entre um âmbito experimental e um nível teórico e abstrato. Em ambos os casos essas pontes evidenciam o caráter metafísico das medições: que elas constituem um modo de conectar o concreto com o abstrato ${ }^{42}$. Porém, elas colocam essa questão através de diferentes pontos de partida. Enquanto na abordagem algébrica fica claro que as medições envolvem considerações metafísicas desde um ponto de vista da aplicabilidade do conhecimento matemático - e que está relacionada com a metafísica realizada em filosofia da matemática, a abordagem baseada em modelos torna claro como a prática

42 Esse tema foi colocado no capítulo 2 de CARTWRIGHT, Nancy. The dappled world: a study of the boundaries of science. Cambridge: Cambridge University Press, 1999. 
das medições envolve considerações metafísicas sobre a relação entre definições conceituais e experimentos.

Justificando essa última asserção, entender as medições a partir de uma teorização baseada em modelos atribui a esses modelos o papel de vincular a construção de um experimento com o corpo teórico advindo da metrologia. Como medir é efetuar uma avaliação comparativa entre magnitudes de grandezas, qualquer procedimento de medição pressupõe a escolha de procedimentos e unidades de comparação, os padrões. Determinar quais os melhores procedimentos e as melhores unidades, estabelecendo os padrões de medida, constitui um dos objetivos da metrologia. Por exemplo, é graças à metrologia que hoje pensamos que a unidade padrão de comprimento é o metro, definido por referência à constante física da velocidade da luz como 1/299 792458 segundo. As funções desempenhadas pela padronização na atividade científica são de suma importância, e não serão o foco das nossas análises aqui. $\mathrm{O}$ que nos interessa é que a abordagem baseada em modelos nos auxilia a compreender que há dois níveis nas medições e, por consequência, no estabelecimento dos padrões.

Dessa forma, temos padrões empíricos, procedimentos e objetos físicos adotados como padrões, e abstratos, construções, esquemas e definições teóricas, as quais são utilizadas para a construção dos modelos. Assim, a prática da medição perfaz a construção de uma conexão entre a definição da unidade de magnitude para a grandeza em questão, como a unidade padrão de massa, e um instrumento de medida. Tal conexão é referida na literatura pelo termo "realização", utilizado no sentido de um instrumento realizar a definição, ou seja, ser capaz de fornecer outcomes que satisfaçam aproximadamente essas definições. Porém, emerge desse cenário a dúvida sobre, afinal, quando e se de fato algo concreto pode satisfazer uma definição desse tipo, por exemplo, quando saberemos que uma mesa exibe/possui algo como "um metro".

Sintetizando, de um ponto de vista metafísico, utilizar modelos como pontes entre níveis abstratos e concretos recai em na questão semelhante 
sobre a utilização de estruturas relacionais qualitativas como ponte entre relações empíricas e numéricas apresentada anteriormente: o que legitima a conexão desses esquemas conceituais com a natureza?

\section{Considerações finais}

O presente ensaio teve por objetivo discorrer sobre o caráter metafísico das medições, afirmando que as práticas das medições possuem elementos metafísicos tanto com relação aos seus alvos, as grandezas e as magnitudes, quanto com relação aos seus procedimentos. Como a filosofia da medição é uma subárea recente da filosofia da ciência, pois apenas no último século as medições foram colocadas enquanto principal objeto de estudo, há uma coleção de problemas que foram pouco trabalhados e permanecem em aberto, como a questão da natureza das grandezas.

Ao mesmo tempo, embora as abordagens mais influentes na literatura da área possuam um viés mais epistêmico do que metafísico, nos parece latente que uma filosofia da medição trabalha com elementos metafísicos, independentemente da teoria filosófica sobre as medições científicas que adotarmos. Utilizando as abordagens baseadas em modelos e a TRM como casos paradigmáticos na literatura da área, vimos que a compreensão do que seja uma medição em ambos os casos capturam, cada concepção ao seu modo, a noção intuitiva de que medir é um processo que relaciona um âmbito empírico e experimental, com um âmbito abstrato. Ao trabalharem com essa relação, as abordagens em filosofia da medição remontam no milenar problema filosófico sobre como o abstrato se relaciona com o concreto, seja na questão da aplicabilidade do conhecimento matemático ao mundo, seja no vínculo entre conceitos e a prática científica experimental. Como já afirmamos, apesar dessa pergunta pairar no horizonte de qualquer teorização sobre o mundo físico, ela encontra um lugar de destaque na filosofia da medição. Do nosso ponto de vista, observar atentamente essas teorizações a partir de um viés metafísico oferece uma oportunidade de colocar o antigo problema sobre a relação entre o 
concreto e o abstrato em novos caminhos e que tem sido pouco percebida na literatura filosófica.

\section{Referências}

ARENHART, Jonas R. B. Ontological frameworks for scientific theories. Foundations of Science, [s. l.], v. 17, n. 4, p. 339-356, 2012. https://doi. org/10.1007/s10699-012-9288-5

BERKA, Karel. Measurement: its concepts, theories and problems. Dordrecht: D. Reidel Publishing Company, 1983. (Boston Studies in the Philosophy of science, v. 72).

BRIDGMAN, P. W. The logic of modern physics. New York: Macmillan, 1927.

CAMPBELL, Norman Robert. Physics: the elements. London: Cambridge University Press, 1920.

CANTÙ, Paola. The epistemological question of the applicability of mathematics. Journal for the History of Analytical Philosophy, [s. l.], v. 6, n. 3, p. 95-114, 2018. https://doi.org/10.15173/jhap.v6i3.3435

CARTWRIGHT, Nancy. The dappled world: a study of the boundaries of science. Cambridge: Cambridge University Press, 1999. https://doi.org/10.1017/ $\mathrm{CBO}_{9781139167093}$

CHANG, Hasok; CARTWRIGHT, Nancy. Measurement. In: PSILLOS, Stathis; CURD, Martin (ed.). The Routledge companion to philosophy of science. 2nd ed. New York: Routledge, 2013. p. 411-419.

CHANG, Hasok. Inventing temperature: measurement and scientific progress. Oxford: Oxford University Press, 2007. (Oxford Studies in Philosophy of Science). https://doi.org/10.1017/s0007087406289052

DÍEZ, José A. A hundred years of numbers. An historical introduction to measurement theory 1887-1990: Part I: The formation period. Two lines of research: Axiomatics and real morphisms, scales and invariance. Studies in History and Philosophy of Science Part A, [s. l.], v. 28, n. 1, p. 167-185, $1997 \mathrm{a}$. https://doi.org/10.1016/s0039-3681(96)00014-3

DÍEZ, José A. A Hundred Years of Numbers. An historical introduction to measurement theory 1887-1990: Part 2: Suppes and the mature theory. Representation and uniqueness. Studies in History and Philosophy of Science 
Part A, [s. l.], v. 28, n. 2, p. 237-265, 1997b. https://doi.org/10.1016/so039$3681(96) 00015-5$

FRIGERIO, Aldo; GIORDANI, Alessandro; MARI, Lucas. Outline of a general model of measurement. Synthese, Dordrecht, v. 175, n. 2, p. 123-149, July 2010. https://doi.org/10.1007/s11229-009-9466-3

HEILMANN, Conrad. A new interpretation of the representational theory of measurement. Philosophy of Science, Baltimore, v. 82, n. 5, p. 787-797, Dec. 2015. https://doi.org/10.1086/683280

HELMHOLTZ, H. Numbering and Measuring from an epistemological viewpoint. In: COHEN, Robert S.; ELKANA, Y. (ed.). Hermann von Helmholtz epistemological writings: the Paul Hertz/Moritz Schlick Centenary Edition of 1921. [S. l.]: Springer, 1977. p. 72-102. (Boston studies in the philosophy of science, v. 37). https://doi.org/10.1007/978-94-010-1115-0 3

KRANTZ, David H. et al. Foundations of measurement. London: Academic Press, 1971. (Additive and Polynomial Representations, v. 1).

KRANTZ, David H. et al. Foundations of measurement. London: Academic Press, 1989. (Geometrical, Threshold and Probabilistic Representations, v. 2).

KRANTZ, David H. et al. Foundations of measurement. London: Academic Press, 1990. (Representation, Axiomatization and Invariance, v. 3).

KRAUSE, Décio. Tópicos em ontologia analítica. São Paulo: UNESP, 2017.

KYBURG, Henry E. Theory and measurement. Cambridge: Cambridge University Press, 1984.

LUCE, D.; SUPPES, P. Representational measurement theory. In: PASHLER, Hal. (ed.). Steven's handbook of experimental psychology. 3rd ed. New York: John Wiley \& Sons, 2002. p. 1-42. https://doi.org/10.1002/0471214426.pas0401

MARI, Luca. Epistemology of measurement. Measurement, Amsterdam, v. 34, n. 1, p. 17-30, 2003. https://doi.org/10.1016/S0263-2241(03)00016-2

MICHELL, J. Making the representational theory of measurement. In: MICHELL, Joel. Measurement in psychology: a critical history of a methodological concept. Cambridge: Cambridge University Press, 1999. p. 109-139. https://doi. org/10.1017/cbog780511490040.006

NARENS, Louis. Abstract measurement theory. Cambridge: MIT Press, 1985.

STEVENS, S. S. On the theory of scales of measurement. Science, [s. l.], v. 103 , n. 2684, p. 677-680, June 1946. https://doi.org/10.1126/science.103.2684.677 
SUPPES, Patrick. A set of independent axioms for extensive quantities. Portugaliæ Mathematica, Lisboa, v. 10, n. 4, p. 163-172, 1951.

TAL, Eran. Measurement in science. In: ZALTA, Edward N. (ed.). The Stanford Encyclopedia of Philosophy. Stanford: Center for the Study of Language and Information, 2015. Disponível em: https://plato.stanford.edu/entries/ measurement-science. Acesso em: 04 abr. 2019. https://doi.org/10.1108/ rr-06-2015-0155

TAL, Eran. Old and new problems in philosophy of measurement. Philosophy Compass, [s. l.], v. 8, n. 12, p. 1159-1173, 2013. https://doi.org/10.1111/phc3.12089 